Research Article

\title{
Double Controlled Partial Metric Type Spaces and Convergence Results
}

\author{
Haroon Ahmad $\mathbb{D}^{1},{ }^{1}$ Mudasir Younis $\mathbb{D}^{2},{ }^{2}$ and Mehmet Emir Köksal $\mathbb{D i D}^{3}$ \\ ${ }^{1}$ Department of Mathematics, University of Management and Technology, Lahore 54770, Pakistan \\ ${ }^{2}$ Department of Mathematics, Jammu and Kashmir Institute of Mathematical Sciences, Srinagar 190008, \\ Jammu and Kashmir, India \\ ${ }^{3}$ Department of Mathematics, Ondokuz Mayis University, 55139 Atakum, Samsun, Turkey
}

Correspondence should be addressed to Mehmet Emir Köksal; mekoksal@omu.edu.tr

Received 25 October 2021; Accepted 18 November 2021; Published 14 December 2021

Academic Editor: Sun Young Cho

Copyright (c) 2021 Haroon Ahmad et al. This is an open access article distributed under the Creative Commons Attribution License, which permits unrestricted use, distribution, and reproduction in any medium, provided the original work is properly cited.

\begin{abstract}
In this paper, we firstly propose the notion of double controlled partial metric type spaces, which is a generalization of controlled metric type spaces, partial metric spaces, and double controlled metric type spaces. Secondly, our aim is to study the existence of fixed points for Kannan type contractions in the context of double controlled partial metric type spaces. The proposed results enrich, theorize, and sharpen a multitude of pioneer results in the context of metric fixed point theory. Additionally, we provide numerical examples to illustrate the essence of our obtained theoretical results.
\end{abstract}

\section{Introduction and Preliminaries}

The study of fixed points of given mappings satisfying certain contractive conditions in various abstract spaces has been at the middle of vigorous research activity. Banach contraction mapping principle has attracted the eye of the many authors to generalize, extend, and improve the metric fixed point theory. For this purpose, the authors considered the extension of metric fixed point theory to different abstract spaces such as symmetric spaces, quasimetric spaces, fuzzy metric spaces, partial metric spaces, probabilistic metric spaces, and spaces with graph.

The notion of $b$-metric spaces was first presented by Bakhtin [1] and Czerwik [2]. Many writers have since obtained a number of fixed point solutions in $b$-metric spaces for single and multivalued operators. We reference Kamran et al. [3] (see also $[4,5]$ ), who presented extended $b$-metric spaces by manipulating the triangle inequality rather than utilizing control functions, as one of the generalizations concerning $b$-metric spaces. Following that, in 2018, Abdeljawad et al. [6,7] established the concepts of controlled metric type spaces and double controlled metric type spaces, respectively. Souayah and Mrad [8] proposed a more broad idea of controlled partial metric type spaces in 2019. It is useful to establish the extensions of the contraction principle from metric spaces to $b$-metric spaces, and therefore the controlled metric type of spaces is useful to prove the existence and uniqueness of theorems for many forms of integral and differential equations. Some interesting applications can be found in the recent papers [4, 9-15]. It is always interesting to find novel applications dealing with engineering science and technology using fixed point technique.

On the other hand, the notion of partial metric space was given by Matthews $[16,17]$ in 1992, which is the generalization of the usual metric space in which $d(x, x)$ is not zero. After that, many researchers worked on the partial metric type spaces to discover the existence of fixed point and their uniqueness. In 2019, Gu and Shatanawi [18] expounded some coupled fixed point theorems in the context of partial metric spaces for hybrid pairs of mappings satisfying a symmetric type contraction. In 2020, Nguyen and Tram [19] demonstrated various fixed point results involving involution mappings. Recently, in 2021, Javaid et al. [20] 
propounded fixed point results in the setting orthogonal partial metric spaces with application. Researchers can refer to $[14,21-23]$ for further information on fixed points in partial type metric spaces.

Taking into consideration the facts mentioned above, in this article, we introduce the concept of double controlled partial metric type space, which is an extension of the controlled metric type spaces, double controlled metric type spaces, and controlled partial metric type spaces. We also look into the existence and uniqueness of fixed point results, which are Kannan contractions' extensions.

Let us begin by reviewing the definition of double controlled metric space as follows.

Definition 1 (see [6]). Let $X$ be a nonempty set and consider the functions $\alpha, \mu: X \times X \longrightarrow[1, \infty)$.

Let $d: X \times X \longrightarrow[0, \infty)$ satisfy

(1) $d\left(x_{1}, x_{2}\right)=0$ if and only if $x_{1}=x_{2}$,

(2) $d\left(x_{1}, x_{2}\right)=d\left(x_{2}, x_{1}\right)$,

(3) $d\left(x_{1}, x_{2}\right) \leq \alpha\left(x_{1}, x_{3}\right) d\left(x_{1}, x_{3}\right)+\mu\left(x_{3}, x_{2}\right) d\left(x_{3}, x_{2}\right)$, for all $x_{1}, x_{2}, x_{3} \in X$, then $(X, d)$ is called a double controlled metric type space.

\section{Double Controlled Partial Metric Type Spaces}

The following is the formal definition of the double controlled partial metric type space which generalizes the notation of controlled metric type spaces, double controlled metric type spaces, and partial metric spaces.

Definition 2. Let $X$ be a nonempty set consider $\alpha, \mu$ : $X \times$ $X \longrightarrow[1, \infty)$ be a function.

Let $d: X \times X \longrightarrow[0, \infty)$ satisfy

(1) $d\left(x_{1}, x_{2}\right)=0$ if and only if $x_{1}=x_{2}$,

(2) $d\left(x_{1}, x_{2}\right)=d\left(x_{2}, x_{1}\right)$,

(3) $d\left(x_{1}, x_{1}\right) \leq d\left(x_{1}, x_{2}\right)$,

(4) $d\left(x_{1}, x_{2}\right) \leq \alpha\left(x_{1}, x_{3}\right) d\left(x_{1}, x_{3}\right)+\mu\left(x_{3}, x_{2}\right) d\left(x_{3}, x_{2}\right)$, for all $x_{1}, x_{2}, x_{3} \in X$, then $(X, d)$ is called a double controlled partial metric type space.

Note that double controlled partial metric type space is more extensive than the double controlled metric type space.

Example 1. A double controlled partial metric type space is not necessarily a double controlled metric type space.

Let $X=\{0,1,2,3,4\}$ and take $d: X \times X \longrightarrow[0, \infty)$. Consider $\alpha, \mu: X \times X \longrightarrow[1, \infty)$, where

$$
\begin{aligned}
& \alpha(x, y)=d(x, y)+5, \\
& \mu(x, y)=d(x, y)+7 .
\end{aligned}
$$

Let the metric $d$ be defined by the following (Table 1 ).

It is easy to verify that $(p 1)$ and $(p 2)$ are true.

We prove condition (3) with different cases, that is, $d\left(x_{1}, x_{1}\right) \leq d\left(x_{1}, x_{2}\right)$, for all $x_{1}, x_{2} \in X$ and $x_{1} \neq x_{2}$.
Table 1: Metric $d$ defined in Example 1.

\begin{tabular}{cccccc}
\hline$d$ & 0 & 1 & 2 & 3 & 4 \\
\hline 0 & $1 / 27$ & $1 / 4$ & $1 / 5$ & $1 / 6$ & $1 / 7$ \\
1 & $1 / 4$ & $1 / 28$ & $2 / 7$ & $2 / 9$ & $2 / 11$ \\
2 & $1 / 5$ & $2 / 7$ & $1 / 29$ & $3 / 11$ & $3 / 13$ \\
3 & $1 / 6$ & $2 / 9$ & $3 / 11$ & $1 / 28$ & $4 / 13$ \\
4 & $1 / 7$ & $2 / 11$ & $3 / 13$ & $4 / 13$ & $1 / 27$ \\
\hline
\end{tabular}

Case (i): let $d\left(x_{1}, x_{1}\right)=d(0,0)=(1 / 27), \quad d(0,0)$ $\leq d\left(x_{1}, x_{2}\right)$, satisfied for all $x_{1}, x_{2} \in X$ and $x_{1} \neq x_{2}$.

Case (ii): let $d\left(x_{1}, x_{1}\right)=d(1,1)=(1 / 28), d(1,1)$ $\leq d\left(x_{1}, x_{2}\right)$, satisfied for all $x_{1}, x_{2} \in X$ and $x_{1} \neq x_{2}$

Case (iii): let $d\left(x_{1}, x_{1}\right)=d(2,2)=(1 / 29), d(2,2)$ $\leq d\left(x_{1}, x_{2}\right)$, satisfied for all $l x_{1}, x_{2} \in X$ and $x_{1} \neq x_{2}$.

Case (iv): let $d\left(x_{1}, x_{1}\right)=d(3,3)=(1 / 28), d(3,3)$ $\leq d\left(x_{1}, x_{2}\right)$, satisfied for all $x_{1}, x_{2} \in X$ and $x_{1} \neq x_{2}$.

Case (v): let $d\left(x_{1}, x_{1}\right)=d(4,4)=(1 / 27), \quad d(4,4)$ $\leq d\left(x_{1}, x_{2}\right)$, satisfied for all $x_{1}, x_{2} \in X$ and $x_{1} \neq x_{2}$.

Now, we will prove the property $(p 4)$.

Case (i): to satisfy $d(0,0)$, we have

$$
\begin{aligned}
& d(0,0) \leq \alpha(0,0) d(0,0)+\mu(0,0) d(0,0) \\
& 0.0370 \leq 0.4513 \\
& d(0,0) \leq \alpha(0,1) d(0,1)+\mu(1,0) d(1,0) \\
& 0.0370 \leq 3.1250, \\
& d(0,0) \leq \alpha(0,2) d(0,2)+\mu(2,0) d(2,0) \\
& 0.0370 \leq 2.48, \\
& d(0,0) \leq \alpha(0,3) d(0,3)+\mu(3,0) d(3,0) \\
& 0.0370 \leq 2.0555, \\
& d(0,0) \leq \alpha(0,4) d(0,4)+\mu(4,0) d(4,0) \\
& 0.0370 \leq 1.7551 .
\end{aligned}
$$

Case (ii): now, we have to satisfy $d(0,1)=d(1,0)$ :

$$
\begin{aligned}
d(0,1) & \leq \alpha(0,0) d(0,0)+\mu(0,1) d(0,1) \\
0.25 & \leq 1.9990, \\
d(0,1) & \leq \alpha(0,1) d(0,1)+\mu(1,1) d(1,1) \\
0.25 & \leq 1.5637 \\
d(0,1) & \leq \alpha(0,2) d(0,2)+\mu(2,1) d(2,1) \\
0.25 & \leq 3.1216, \\
d(0,1) & \leq \alpha(0,3) d(0,3)+\mu(3,1) d(3,1) \\
0.25 & \leq 2.4660, \\
d(0,1) & \leq \alpha(0,4) d(0,4)+\mu(4,1) d(4,1) \\
0.25 & \leq 2.0404 .
\end{aligned}
$$

Case (iii): to prove $d(0,2)=d(2,0)$, we have 


$$
\begin{aligned}
d(0,2) & \leq \alpha(0,0) d(0,0)+\mu(0,2) d(0,2) \\
0.2 & \leq 1.6265, \\
d(0,2) & \leq \alpha(0,1) d(0,1)+\mu(1,2) d(1,2) \\
0.2 & \leq 3.3941, \\
d(0,2) & \leq \alpha(0,2) d(0,2)+\mu(2,2) d(2,2) \\
0.2 & \leq 1.2825, \\
d(0,2) & \leq \alpha(0,3) d(0,3)+\mu(3,2) d(3,2) \\
0.2 & \leq 2.8445, \\
d(0,2) & \leq \alpha(0,4) d(0,4)+\mu(4,2) d(4,2) \\
0.2 & \leq 2.4033 .
\end{aligned}
$$

Case (iv): in order to show $d(0,3)=d(3,0)$, we proceed as follows:

$$
\begin{aligned}
& d(0,3) \leq \alpha(0,0) d(0,0)+\mu(0,3) d(0,3) \\
& 0.1666 \leq 1.3810, \\
& d(0,3) \leq \alpha(0,1) d(0,1)+\mu(1,3) d(1,3) \\
& 0.1666 \leq 2.9174, \\
& d(0,3) \leq \alpha(0,2) d(0,2)+\mu(2,3) d(2,3) \\
& 0.1666 \leq 3.0234, \\
& d(0,3) \leq \alpha(0,3) d(0,3)+\mu(3,3) d(3,3) \\
& 0.1666 \leq 1.1123, \\
& d(0,3) \leq \alpha(0,4) d(0,4)+\mu(4,3) d(4,3) \\
& 0.1666 \leq 2.9832 .
\end{aligned}
$$

Case (v): now, we have to satisfy $d(0,4)=d(4,0)$ :

$$
\begin{aligned}
& d(0,4) \leq \alpha(0,0) d(0,0)+\mu(0,4) d(0,4) \\
& 0.1428 \leq 1.2069, \\
& d(0,4) \leq \alpha(0,1) d(0,1)+\mu(1,4) d(1,4) \\
& 0.1428 \leq 2.6182, \\
& d(0,4) \leq \alpha(0,2) d(0,2)+\mu(2,4) d(2,4) \\
& 0.1428 \leq 2.7086, \\
& d(0,4) \leq \alpha(0,3) d(0,3)+\mu(3,4) d(3,4) \\
& 0.1428 \leq 3.1096, \\
& d(0,4) \leq \alpha(0,4) d(0,4)+\mu(4,4) d(4,4) \\
& 0.1428 \leq 0.9953 .
\end{aligned}
$$

Case (vi): for the case $d(1,1)$, we have

$$
\begin{aligned}
d(1,1) & \leq \alpha(1,0) d(1,0)+\mu(0,1) d(0,1) \\
0.03571 & \leq 3.125, \\
d(1,1) & \leq \alpha(1,1) d(1,1)+\mu(1,1) d(1,1) \\
0.03571 & \leq 0.4311, \\
d(1,1) & \leq \alpha(1,2) d(1,2)+\mu(2,1) d(2,1)
\end{aligned}
$$

$0.03571 \leq 3.5918$,

$$
\begin{aligned}
d(1,1) & \leq \alpha(1,3) d(1,3)+\mu(3,1) d(3,1) \\
0.03571 & \leq 2.7654, \\
d(1,1) & \leq \alpha(1,4) d(1,4)+\mu(1,4) d(4,1) \\
0.03571 & \leq 2.2479 .
\end{aligned}
$$

Case (vii): to satisfy $d(1,2)=d(2,1)$, we have

$$
\begin{aligned}
& d(1,2) \leq \alpha(1,0) d(1,0)+\mu(0,2) d(0,2) \\
& 0.2857 \leq 2.7525, \\
& d(1,2) \leq \alpha(1,1) d(1,1)+\mu(1,2) d(1,2) \\
& 0.2857 \leq 2.2614, \\
& d(1,2) \leq \alpha(1,2) d(1,2)+\mu(2,2) d(2,2) \\
& 0.2857 \leq 1.7527, \\
& d(1,2) \leq \alpha(1,3) d(1,3)+\mu(3,2) d(3,2) \\
& 0.2857 \leq 3.1439, \\
& d(1,2) \leq \alpha(1,4) d(1,4)+\mu(4,2) d(4,2) \\
& 0.2857 \leq 2.6107 .
\end{aligned}
$$

Case (viii): now, we have to satisfy $d(1,3)=d(3,1)$ :

$$
\begin{aligned}
& d(1,3) \leq \alpha(1,0) d(1,0)+\mu(0,3) d(0,3) \\
& 0.2222 \leq 2.5069, \\
& d(1,3) \leq \alpha(1,1) d(1,1)+\mu(1,3) d(1,3) \\
& 0.2222 \leq 1.7847, \\
& d(1,3) \leq \alpha(1,2) d(1,2)+\mu(2,3) d(2,3) \\
& 0.2222 \leq 3.4936, \\
& d(1,3) \leq \alpha(1,3) d(1,3)+\mu(3,3) d(3,3) \\
& 0.2222 \leq 1.4117, \\
& d(1,3) \leq \alpha(1,4) d(1,4)+\mu(4,3) d(4,3) \\
& 0.2222 \leq 3.19066 .
\end{aligned}
$$

Case (ix): for the case $d(1,4)=d(4,1)$, consider the following:

$$
\begin{aligned}
& d(1,4) \leq \alpha(1,0) d(1,0)+\mu(0,4) d(0,4) \\
& 0.1818 \leq 2.3329, \\
& d(1,4) \leq \alpha(1,1) d(1,1)+\mu(1,4) d(1,4) \\
& 0.1818 \leq 1.4856, \\
& d(1,4) \leq \alpha(1,2) d(1,2)+\mu(2,4) d(2,4) \\
& 0.1818 \leq 3.1788, \\
& d(1,4) \leq \alpha(1,3) d(1,3)+\mu(3,4) d(3,4) \\
& 0.1818 \leq 3.4090, \\
& d(1,4) \leq \alpha(1,4) d(1,4)+\mu(4,4) d(4,4) \\
& 0.1818 \leq 1.2027 .
\end{aligned}
$$


Case $(\mathrm{x})$ : for the case $d(2,2)$, we have

$$
\begin{aligned}
& d(2,2) \leq \alpha(2,0) d(2,0)+\mu(0,2) d(0,2) \\
& 0.0344 \leq 2.48, \\
& d(2,2) \leq \alpha(2,1) d(2,1)+\mu(1,2) d(1,2) \\
& 0.0344 \leq 3.5918, \\
& d(2,2) \leq \alpha(2,2) d(2,2)+\mu(2,2) d(2,2) \\
& 0.0344 \leq 0.4161, \\
& d(2,2) \leq \alpha(2,3) d(2,3)+\mu(2,3) d(2,3) \\
& 0.0344 \leq 3.4214, \\
& d(2,2) \leq \alpha(2,4) d(2,4)+\mu(4,2) d(4,2) \\
& 0.0344 \leq 2.8757 .
\end{aligned}
$$

Case (xi): to satisfy $d(2,3)=d(3,2)$, we proceed as follows:

$$
\begin{aligned}
& d(2,3) \leq \alpha(2,0) d(2,0)+\mu(0,3) d(0,3) \\
& 0.2727 \leq 2.2344 \\
& d(2,3) \leq \alpha(2,1) d(2,1)+\mu(1,3) d(1,3) \\
& 0.2727 \leq 3.1151, \\
& d(2,3) \leq \alpha(2,2) d(2,2)+\mu(2,3) d(2,3) \\
& 0.2727 \leq 2.1570, \\
& d(2,3) \leq \alpha(2,3) d(2,3)+\mu(3,3) d(3,3) \\
& 0.2727 \leq 1.6892, \\
& d(2,3) \leq \alpha(2,4) d(2,4)+\mu(4,3) d(4,3) \\
& 0.2727 \leq 3.4556 .
\end{aligned}
$$

Case (xii): next, we have to satisfy $d(2,4)=d(4,2)$ :

$$
\begin{aligned}
& d(2,4) \leq \alpha(2,0) d(2,0)+\mu(0,4) d(0,4) \\
& 0.2307 \leq 2.0604 \\
& d(2,4) \leq \alpha(2,1) d(2,1)+\mu(1,4) d(1,4) \\
& 0.2307 \leq 2.8159 \\
& d(2,4) \leq \alpha(2,2) d(2,2)+\mu(2,4) d(2,4) \\
& 0.2307 \leq 1.8422 \\
& d(2,4) \leq \alpha(2,3) d(2,3)+\mu(3,4) d(3,4) \\
& 0.2307 \leq 3.6865, \\
& d(2,4) \leq \alpha(2,4) d(2,4)+\mu(4,4) d(4,4) \\
& 0.2307 \leq 1.4677 .
\end{aligned}
$$

Case(xiii): now, for the case $d(3,3)$, we consider

$$
\begin{aligned}
d(3,3) & \leq \alpha(3,0) d(3,0)+\mu(0,3) d(0,3) \\
0.03571 & \leq 2.0555, \\
d(3,3) & \leq \alpha(3,1) d(3,1)+\mu(1,3) d(1,3) \\
0.03571 & \leq 2.7654,
\end{aligned}
$$

$$
\begin{aligned}
d(3,3) & \leq \alpha(3,2) d(3,2)+\mu(2,3) d(2,3) \\
0.03571 & \leq 3.4214 \\
d(3,3) & \leq \alpha(3,3) d(3,3)+\mu(3,3) d(3,3) \\
0.03571 & \leq 0.4311, \\
d(3,3) & \leq \alpha(3,4) d(3,4)+\mu(4,3) d(4,3) \\
0.03571 & \leq 3.8816 .
\end{aligned}
$$

Case (xiv): now, we have to satisfy $d(3,4)=d(4,3)$ :

$$
\begin{aligned}
& d(3,4) \leq \alpha(3,0) d(3,0)+\mu(0,4) d(0,4) \\
& 0.3076 \leq 1.8855, \\
& d(3,4) \leq \alpha(3,1) d(3,1)+\mu(1,4) d(1,4) \\
& 0.3076 \leq 2.4662, \\
& d(3,4) \leq \alpha(3,2) d(3,2)+\mu(2,4) d(2,4) \\
& 0.3076 \leq 3.1066, \\
& d(3,4) \leq \alpha(3,3) d(3,3)+\mu(3,4) d(3,4) \\
& 0.3076 \leq 2.4283, \\
& d(3,4) \leq \alpha(3,4) d(3,4)+\mu(4,4) d(4,4) \\
& 0.3076 \leq 1.8937 .
\end{aligned}
$$

Case (xv): lastly, for the case $d(4,4)$, we have

$$
\begin{aligned}
& d(4,4) \leq \alpha(4,0) d(4,0)+\mu(0,4) d(0,4) \\
& 0.0370 \leq 1.7551, \\
& d(4,4) \leq \alpha(4,1) d(4,1)+\mu(1,4) d(1,4) \\
& 0.0370 \leq 2.2479, \\
& d(4,4) \leq \alpha(4,2) d(4,2)+\mu(2,4) d(2,4) \\
& 0.0370 \leq 2.8757, \\
& d(4,4) \leq \alpha(4,3) d(4,3)+\mu(3,4) d(3,4) \\
& 0.0370 \leq 3.8816, \\
& d(4,4) \leq \alpha(4,4) d(4,4)+\mu(4,4) d(4,4) \\
& 0.0370 \leq 0.4471 .
\end{aligned}
$$

Therefore, $(X, d)$ is a double controlled partial metric type space but is not a double controlled metric type space since $d(x, x)$ is not equal to zero all the time.

We define Cauchy and convergent sequence in double controlled partial metric type spaces as follows.

Definition 3. Let $(X, d)$ be a double controlled partial metric type space; the sequence $\left\{x_{n}\right\}_{n \geq 0}$ converges to some $x$ in $X$, if $\lim _{n, m \rightarrow \infty} d\left(x_{n}, x\right)=d(x, x)$; in this case, we write $\lim _{n \rightarrow \infty} x_{n}=x$.

Definition 4. The sequence $\left\{x_{n}\right\}$ in a double controlled partial metric type space $(X, d)$ is said to be Cauchy sequence, if $\lim _{n, m \longrightarrow \infty} d\left(x_{n}, x_{m}\right)$ exists and is finite. 
Definition 5. A double controlled partial metric type space $(X, d)$ is said to be complete if every Cauchy sequence $x$ in $X$ converges to a point $x \in X$, that is, $d(x, x)=\lim _{n, m \longrightarrow \infty} d\left(x_{n}, x_{m}\right)$.

Definition 6. Let $(X, d)$ be a double controlled partial metric type space. Let $x \in X$ and $\varepsilon>0$.

(i) The open ball $B_{p}(x, \varepsilon)$ is

$$
B_{p}(x, \varepsilon)=\{y \in X, d(x, y)<d(x, x)+\varepsilon\} .
$$

(ii) The mapping $T: X \longrightarrow X$ is said to be continuous at $x \in X$ if for all $\varepsilon>0$, there exists $\delta>0$ such that

$$
T\left(B_{p}(x, \delta)\right) \subseteq B_{p}(T x, \varepsilon) .
$$

Therefore, if $T$ is continuous at $x$ in the double controlled partial metric type space $(X, d)$, then $x_{n} \longrightarrow x$ implies that $T x_{n} \longrightarrow T x$ as $n \longrightarrow \infty$

\section{Some Novel Results}

This section is devoted to discuss some fixed point results in double controlled partial metric type space $(X, d)$. The main result of this article is given by the following theorem.

Theorem 1. Let $(X, d)$ be a complete double controlled partial metric type space by the functions $\alpha, \mu: X \times X \longrightarrow[1, \infty)$. Suppose that $f: X \longrightarrow X$ satisfies

$$
d(f x, f y) \leq \beta[d(x, f x)+(y, f y)]
$$

for all $x, y \in X$, where $\beta \in(0,(1 / 2))$. For $x_{0} \in X$, take $x_{n}=f^{n} x_{0}$, assuming that

$$
\sup _{m \geq 1} \lim _{i \longrightarrow \infty} \frac{\alpha\left(x_{i+1}, x_{i+2}\right)}{\alpha\left(x_{i}, x_{i+1}\right)} \mu\left(x_{i}, x_{m}\right)<\frac{1}{k}, \quad \text { where } k \in(0,1) \text {. }
$$

Furthermore, assume that for every $x \in X$, $\lim _{n \rightarrow \infty} \alpha\left(x, x_{n}\right), \lim _{n \rightarrow \infty} \alpha\left(x_{n}, x\right), \lim _{n \rightarrow \infty} \mu\left(x, x_{n}\right)$, and $\lim _{n \rightarrow \infty} \mu\left(x_{n}, x\right)$ exist and are finite. Then, the sequence $\left\{x_{n}\right\}$ converges to some $u \in X$; moreover, if $\alpha$ and $\mu$ satisfy the following assumptions,

$$
\lim _{n \longrightarrow \infty} \frac{\alpha\left(u, x_{n+1}\right)}{1-\beta \mu\left(x_{n+1}, f u\right)} \leq 0,
$$

then $f$ has a unique fixed point.

Proof. Consider $x_{n}=f^{n} x_{0}$, let $x_{1} \in X$ be arbitrary, and let $x_{2}=f x_{1}$ and let $x_{3}=f x_{2}$ be chosen.

By using (19), we get

$$
\begin{aligned}
d\left(x_{2}, x_{3}\right) & =d\left(f x_{1}, f x_{2}\right) \leq \beta\left[d\left(x_{1}, f x_{1}\right)+d\left(x_{2}, f x_{2}\right)\right] \\
& =\beta\left[d\left(x_{1}, x_{2}\right)+d\left(x_{2}, x_{3}\right)\right] .
\end{aligned}
$$

Then,

$d\left(x_{2}, x_{3}\right) \leq \frac{\beta}{1-\beta} d\left(x_{1}, x_{2}\right), \quad$ where $\frac{\beta}{1-\beta}=\eta \in[0,1)$. obtain

By repeating the same procedure in inequality (23), we

$$
d\left(x_{n}, x_{n+1}\right) \leq \eta^{n-1} d\left(x_{1}, x_{2}\right)
$$

Now, we have to show that $\left\{x_{n}\right\}$ is Cauchy sequence. Since $(X, d)$ is a double controlled partial metric type space, for all natural numbers $n, m \in N$ with $n<m$, we acquire

$$
\begin{aligned}
d\left(x_{n}, x_{m}\right) \leq & \alpha\left(x_{n}, x_{n+1}\right) d\left(x_{n}, x_{n+1}\right)+\mu\left(x_{n+1}, x_{m}\right) d\left(x_{n+1}, x_{m}\right) \\
\leq & \alpha\left(x_{n}, x_{n+1}\right) d\left(x_{n}, x_{n+1}\right)+\alpha\left(x_{n+1}, x_{n+2}\right) \mu\left(x_{n+1}, x_{m}\right) d\left(x_{n+1}, x_{n+2}\right) \\
& +\mu\left(x_{n+1}, x_{m}\right) \mu\left(x_{n+2}, x_{m}\right) d\left(x_{n+2}, x_{m}\right) \\
\leq & \alpha\left(x_{n}, x_{n+1}\right) d\left(x_{n}, x_{n+1}\right)+\alpha\left(x_{n+1}, x_{n+2}\right) \mu\left(x_{n+1}, x_{m}\right) d\left(x_{n+1}, x_{n+2}\right) \\
& +\alpha\left(x_{n+2}, x_{n+3}\right) \mu\left(x_{n+1}, x_{m}\right) \mu\left(x_{n+2}, x_{m}\right) d\left(x_{n+2}, x_{n+3}\right) \\
& +\mu\left(x_{n+1}, x_{m}\right) \mu\left(x_{n+2}, x_{m}\right) \mu\left(x_{n+3}, x_{m}\right) d\left(x_{n+3}, x_{m}\right) \\
\leq & \alpha\left(x_{n}, x_{n+1}\right) d\left(x_{n}, x_{n+1}\right)+\sum_{i=n+1}^{m-2}\left(\prod_{j=n+1}^{i} \mu\left(x_{j}, x_{m}\right)\right) \alpha\left(x_{i}, x_{i+1}\right) d\left(x_{i}, x_{i+1}\right) \\
& +\prod_{k=n+1}^{m-1} \mu\left(x_{k}, x_{m}\right) d\left(x_{m-1}, x_{m}\right)
\end{aligned}
$$




$$
\begin{aligned}
\leq & \alpha\left(x_{n}, x_{n+1}\right) \eta^{n} d\left(x_{0}, x_{1}\right)+\sum_{i=n+1}^{m-2}\left(\prod_{j=n+1}^{i} \mu\left(x_{j}, x_{m}\right)\right) \alpha\left(x_{i}, x_{i+1}\right) \eta^{i} d\left(x_{0}, x_{1}\right) \\
& +\prod_{k=n+1}^{m-1} \mu\left(x_{k}, x_{m}\right) \eta^{m-1} d\left(x_{0}, x_{1}\right) \\
& +\prod_{k=n+1}^{m-1} \mu\left(x_{k}, x_{m}\right) \alpha\left(x_{m-1}, x_{m}\right) \eta^{m-1} d\left(x_{0}, x_{1}\right) \\
= & \alpha\left(x_{n}, x_{n+1}\right) \eta^{n} d\left(x_{0}, x_{1}\right)+\sum_{i=n+1}^{m-1}\left(\prod_{j=n+1}^{i} \mu\left(x_{j}, x_{m}\right)\right) \alpha\left(x_{i}, x_{i+1}\right) \eta^{i} d\left(x_{0}, x_{1}\right) \\
\leq & \alpha\left(x_{n}, x_{n+1}\right) \eta^{n} d\left(x_{0}, x_{1}\right)+\sum_{i=n+1}^{m-1}\left(\prod_{j=n+1}^{i} \mu\left(x_{j}, x_{m}\right)\right) \alpha\left(x_{i}, x_{i+1}\right) \eta^{i} d\left(x_{0}, x_{1}\right) \\
\leq & \alpha\left(x_{n}, x_{n+1}\right) \eta^{n} d\left(x_{0}, x_{1}\right)+\sum_{i=n+1}^{m-1}\left(\prod_{j=n+1}^{i} \mu\left(x_{j}, x_{m}\right)\right) \alpha\left(x_{i}, x_{i+1}\right) \eta^{i} d\left(x_{0}, x_{1}\right) .
\end{aligned}
$$

Assume that

$$
S_{p}=\sum_{i=n+1}^{m-1}\left(\prod_{j=0}^{i} \mu\left(x_{j}, x_{m}\right)\right) \alpha\left(x_{i}, x_{i+1}\right) \eta^{i} d\left(x_{0}, x_{1}\right) \text {. }
$$

Then, we obtain

$$
d\left(x_{n}, x_{m}\right) \leq d\left(x_{0}, x_{1}\right)\left[\eta^{n} \alpha\left(x_{n}, x_{n+1}\right)+\left(S_{m-1}-S_{n}\right)\right] .
$$

Using ratio test, we have

$$
a_{i}=\left(\prod_{j=0}^{i} \mu\left(x_{j}, x_{m}\right)\right) \alpha\left(x_{i}, x_{i+1}\right) \eta^{i} d\left(x_{0}, x_{1}\right), \quad \text { where } \frac{a_{i+1}}{a_{i}}<\frac{1}{\eta} \text {. }
$$

Taking $\lim _{n, m \rightarrow \infty}$, (27) becomes

$$
\lim _{n, m \longrightarrow \infty} d\left(x_{n}, x_{m}\right)=0 \text {. }
$$

This implies that $\left\{x_{n}\right\}$ is a Cauchy sequence in a complete double controlled metric type space $(X, d)$, so $\left\{x_{n}\right\}$ converges to some $u \in X$. Now, we have to prove that $u$ is a fixed point of $T$, so we need to verify that

$$
d(u, f u)=d(u, u)=d(f u, f u) .
$$

From the ( $p 3)$, we have

$$
\begin{array}{r}
d(u, u) \leq d(u, f u), \\
d(f u, f u) \leq d(u, f u) .
\end{array}
$$

Hence, for proving $f u=u$, it is sufficient to prove that $d(u, u) \geq d(u, f u)$ and $d(f u, f u) \geq d(u, f u)$. The triangular inequality yields that

$$
\begin{aligned}
d(u, f u) \leq & \alpha\left(u, x_{n+1}\right) d\left(u, x_{n+1}\right)+\mu\left(x_{n+1}, f u\right) d\left(x_{n+1}, f u\right) \\
\leq & \alpha\left(u, x_{n+1}\right) d\left(u, x_{n+1}\right)+\mu\left(x_{n+1}, f u\right) d\left(f x_{n}, f u\right) \\
\leq & \alpha\left(u, x_{n+1}\right) d\left(u, x_{n+1}\right)+\beta \mu\left(x_{n+1}, f u\right) d\left(x_{n}, f x_{n}\right) \\
& +\beta \mu\left(x_{n+1}, f u\right) d(u, f u) .
\end{aligned}
$$

Taking limit as $n \longrightarrow \infty$, we obtain

$$
d(u, f u) \leq \lim _{n \longrightarrow \infty} \frac{\alpha\left(u, x_{n+1}\right)}{1-\beta \mu\left(x_{n+1}, f u\right) d(u, f u)} d(u, u) .
$$

Utilizing condition (21), we get

$$
d(u, f u) \leq d(u, u)
$$

On the other hand,

$$
\begin{aligned}
d(u, f u) \leq & \alpha(u, f u) d(u, f u)+\mu(f u, f u) d(f u, f u) \\
\leq & \alpha(u, f u) d(u, f u) \\
& +\mu(f u, f u) \beta[d(u, f u)+d(u, f u)] \\
\leq & \alpha(u, f u) d(u, f u)+\beta \mu(f u, f u) d(u, f u) \\
& +\beta \mu(f u, f u) d(u, f u) \\
\leq & \frac{\alpha(u, f u)}{1-\beta \mu(f u, f u)} d(f u, f u) .
\end{aligned}
$$

Hence, we get

$$
d(u, f u) \leq d(u, u)
$$

From (31)-(36), we obtain

$$
u=f u \text {. }
$$

Uniqueness: assume that there are two fixed points $u$ and $v$ of $T$, then

$$
\begin{aligned}
d(u, v) & =d(f u, f v) \leq \beta[d(u, f u)+d(v, f v)] \\
& =\beta[d(u, u)+d(v, v)]
\end{aligned}
$$

Furthermore, we have 


$$
d(u, u)=d(f u, f u) \leq 2 \beta d(u, f u)=2 \beta d(u, u),
$$

where $\beta>1$, then $d(u, u)=0$, similarly

$$
d(v, v)=d(f v, f v) \leq 2 \beta d(v, f v)=2 \beta d(v, v) .
$$

Then, $d(v, v)=0$. Since $d(u, u)=d(v, v)=0$, then $d(u, v)=0$. Therefore, $d(u, u)=d(v, v)=d(u, v)$, which gives $u=v$ and $T$ has a unique fixed point.

Definition 7. Let $(X, d)$ be complete double controlled partial type metric space; a mapping $T: X \longrightarrow X$ is sequentially convergent. For every sequence $\left\{x_{n}\right\}$, if $\left\{f x_{n}\right\}$ is convergent, then $\left\{x_{n}\right\}$ also converges. Also, $f$ is said to be subsequentially convergent. For every sequence $\left\{x_{n}\right\}$, if $\left\{f x_{n}\right\}$ is convergent, then $\left\{x_{n}\right\}$ has a convergent subsequence.

Theorem 2. Let $(X, d)$ be a complete double controlled partial metric type space and $f, g: X \longrightarrow X$ be mapping such that $f$ is continuous, one-to-one, and subsequentially convergent

$$
d(f g x, f g y) \leq \beta[d(f x, f g x)+(f y, f g y)]
$$

For all $x, y \in X$, where $\beta \in(0,(1 / 2))$. For $x_{0} \in X$, take $x_{n}=g^{n} x_{0}$, assuming that

$$
\sup _{m \geq 1} \lim _{i \longrightarrow \infty} \frac{\alpha\left(f x_{i+1}, f x_{i+2}\right)}{\alpha\left(f x_{i}, f x_{i+1}\right)} \mu\left(f x_{i}, f x_{m}\right)<\frac{1}{k}, \quad \text { where } k \in(0,1) \text {. }
$$

Furthermore, assume that for every $x \in X$, $\lim _{n \rightarrow \infty} \alpha\left(x, x_{n}\right), \lim _{n \longrightarrow \infty} \alpha\left(x_{n}, x\right), \lim _{n \longrightarrow \infty} \mu\left(x, x_{n}\right)$, and $\lim _{n \rightarrow \infty} \mu\left(x_{n}, x\right)$ exist and are finite. Then, $g$ has a unique fixed point.

Proof. Let $x_{0}$ be an arbitrary point in $X$ and consider the sequence $\left\{x_{n}\right\}$ defined in the hypothesis of the theorem. From (41), we obtain

$$
\begin{aligned}
d\left(f x_{n}, f x_{n+1}\right) & =d\left(f g x_{n-1}, f x_{n}\right) \\
& \leq \beta\left[d\left(f x_{n-1}, f g x_{n-1}\right)+d\left(f x_{n}, f g x_{n}\right)\right] \\
& =\beta\left[d\left(f x_{n-1}, f g x_{n-1}\right)+d\left(f x_{n}, f x_{n+1}\right)\right] \\
& =\frac{\beta}{1-\beta} d\left(f x_{n-1}, f g x_{n-1}\right) .
\end{aligned}
$$

By induction, we get

$$
d\left(f x_{n}, f x_{n+1}\right) \leq\left(\frac{\beta}{1-\beta}\right)^{n} d\left(f g x_{n-1}, f x_{n}\right)=\eta^{n} d\left(f g x_{0}, f x_{1}\right),
$$

where

$$
\frac{\beta}{1-\beta}=\eta \in[0,1) \text {. }
$$

Now, we have to show that $\left\{f x_{n}\right\}$ is a Cauchy sequence. Since $(X, d)$ is double controlled partial metric type space for all natural numbers $n, m \in N$ with $n<m$, we get

$$
\begin{aligned}
d\left(f x_{n}, f x_{m}\right) \leq & \alpha\left(f x_{n}, f x_{n+1}\right) d\left(f x_{n}, f x_{n+1}\right)+\mu\left(f x_{n+1}, f x_{m}\right) d\left(f x_{n+1}, f x_{m}\right) \\
\leq & \alpha\left(f x_{n}, f x_{n+1}\right) d\left(f x_{n}, f x_{n+1}\right)+\alpha\left(f x_{n+1}, f x_{n+2}\right) \mu\left(f x_{n+1}, f x_{m}\right) d\left(f x_{n+1}, f x_{n+2}\right) \\
& +\mu\left(f x_{n+1}, f x_{m}\right) \mu\left(f x_{n+2}, f x_{m}\right) d\left(f x_{n+2}, f x_{m}\right) \\
\leq & \alpha\left(f x_{n}, f x_{n+1}\right) d\left(f x_{n}, f x_{n+1}\right)+\alpha\left(f x_{n+1}, f x_{n+2}\right) \mu\left(f x_{n+1}, f x_{m}\right) d\left(f x_{n+1}, f x_{n+2}\right) \\
& +\alpha\left(f x_{n+2}, f x_{n+3}\right) \mu\left(f x_{n+1}, f x_{m}\right) \mu\left(f x_{n+2}, f x_{m}\right) d\left(f x_{n+2}, f x_{n+3}\right) \\
& +\mu\left(f x_{n+1}, f x_{m}\right) \mu\left(f x_{n+2}, f x_{m}\right) \mu\left(f x_{n+3}, f x_{m}\right) d\left(f x_{n+3}, f x_{m}\right) \\
\leq & \alpha\left(f x_{n}, f x_{n+1}\right) d\left(f x_{n}, f x_{n+1}\right)+\sum_{i=n+1}^{m-2}\left(\prod_{j=n+1}^{i} \mu\left(f x_{j}, f x_{m}\right)\right) \alpha\left(f x_{i}, f x_{i+1}\right) d\left(f x_{i}, f x_{i+1}\right) \\
& +\prod_{k=n+1}^{m-1} \mu\left(f x_{k}, f x_{m}\right) d\left(f x_{m-1}, f x_{m}\right) \\
\leq & \alpha\left(f x_{n}, f x_{n+1}\right) \eta^{n} d\left(f x_{0}, f x_{1}\right)+\sum_{i=n+1}^{m-2}\left(\prod_{j=n+1}^{i} \mu\left(f x_{j}, f x_{m}\right)\right) \alpha\left(f x_{i}, f x_{i+1}\right) \eta^{i} d\left(f x_{0}, f x_{1}\right) \\
& +\prod_{k=n+1}^{m-1} \mu\left(f x_{k}, f x_{m}\right) \eta^{m-1} d\left(f x_{0}, f x_{1}\right)
\end{aligned}
$$




$$
\begin{aligned}
\leq & \alpha\left(f x_{n}, f x_{n+1}\right) \eta^{n} d\left(f x_{0}, f x_{1}\right)+\sum_{i=n+1}^{m-2}\left(\prod_{j=n+1}^{i} \mu\left(f x_{j}, f x_{m}\right)\right) \alpha\left(f x_{i}, f x_{i+1}\right) \eta^{i} d\left(f x_{0}, f x_{1}\right) \\
& +\prod_{k=n+1}^{m-1} \mu\left(f x_{k}, f x_{m}\right) \alpha\left(f x_{m-1}, f x_{m}\right) \eta^{m-1} d\left(f x_{0}, f x_{1}\right) \\
= & \alpha\left(f x_{n}, f x_{n+1}\right) \eta^{n} d\left(f x_{0}, f x_{1}\right)+\sum_{i=n+1}^{m-1}\left(\prod_{j=n+1}^{i} \mu\left(f x_{j}, f x_{m}\right)\right) \alpha\left(f x_{i}, f x_{i+1}\right) \eta^{i} d\left(f x_{0}, f x_{1}\right) \\
\leq & \alpha\left(f x_{n}, f x_{n+1}\right) \eta^{n} d\left(f x_{0}, f x_{1}\right)+\sum_{i=n+1}^{m-1}\left(\prod_{j=n+1}^{i} \mu\left(f x_{j}, f x_{m}\right)\right) \alpha\left(f x_{i}, f x_{i+1}\right) \eta^{i} d\left(f x_{0}, f x_{1}\right) \\
\leq & \alpha\left(f x_{n}, f x_{n+1}\right) \eta^{n} d\left(f x_{0}, f x_{1}\right)+\sum_{i=n+1}^{m-1}\left(\prod_{j=n+1}^{i} \mu\left(f x_{j}, f x_{m}\right)\right) \alpha\left(f x_{i}, f x_{i+1}\right) \eta^{i} d\left(f x_{0}, f x_{1}\right) .
\end{aligned}
$$

Assume that

$S_{p}=\sum_{i=n+1}^{m-1}\left(\prod_{j=0}^{i} \mu\left(f x_{j}, f x_{m}\right)\right) \alpha\left(f x_{i}, f x_{i+1}\right) \eta^{i} d\left(f x_{0}, f x_{1}\right)$.

Then, we obtain

$$
d\left(f x_{n}, f x_{m}\right) \leq d\left(f x_{0}, f x_{1}\right)\left[\eta^{n} \alpha\left(f x_{n}, f x_{n+1}\right)+\left(S_{m-1}-S_{n}\right)\right] .
$$

Using ratio test, we have

$$
\begin{gathered}
a_{i}=\left(\prod_{j=0}^{i} \mu\left(f x_{j}, f x_{m}\right)\right) \alpha\left(f x_{i}, f x_{i+1}\right) \eta^{i} d \\
\left(f x_{0}, f x_{1}\right), \quad \text { where } \frac{a_{i+1}}{a_{i}}<\frac{1}{\eta} .
\end{gathered}
$$

Taking $\lim _{n, m \rightarrow \infty}$ inequality, (48) reduces to

$$
\lim _{n, m \rightarrow \infty} d\left(f x_{n}, f x_{m}\right)=0
$$

This amounts to say that $\left\{f x_{n}\right\}$ is a Cauchy sequence in a complete double controlled partial metric type space $(X, d)$, hence there exists $v \in X$ such that

$$
\lim _{n \rightarrow \infty} f x_{n}=v \text {. }
$$

Since $f$ is convergent, the sequence $\left\{x_{n}\right\}$ has a convergent subsequence denoted by $\left\{x_{n_{k}}\right\}_{k=1}^{\infty}$ such that

$$
\lim _{k \longrightarrow \infty} x_{n_{k}}=u \text {. }
$$

Using the continuity of $f$, we obtain

$$
\lim _{k \longrightarrow \infty} f x_{n_{k}}=f u \text {. }
$$

From (51) and (53), we conclude that $f u=v$. Making use of triangular inequality, we get

$$
\begin{aligned}
d(f g u, f u) \leq & \alpha\left(f g u, f g^{n_{k}} x_{0}\right) d\left(f g u, f g^{n_{k}} x_{0}\right)+\mu\left(f g^{n_{k}} x_{0}, f u\right) d\left(f g^{n_{k}} x_{0}, f u\right) \\
\leq & \alpha\left(f g u, f g^{n_{k}} x_{0}\right) \beta\left[d(f u, f g u)+d\left(f g^{n_{k}-1} x_{0}, f g^{n_{k}} x_{0}\right)\right] \\
& +\mu\left(f g^{n_{k}} x_{0}, f u\right) d\left(f g^{n_{k}} x_{0}, f u\right) \\
\leq & \beta \alpha\left(f g u, f g^{n_{k}} x_{0}\right) d(f u, f g u)+\beta \alpha\left(f g u, f g^{n_{k}} x_{0}\right) d\left(f g^{n_{k}-1} x_{0}, f g^{n_{k}} x_{0}\right) \\
& +\mu\left(f g^{n_{k}} x_{0}, f u\right) d\left(f g^{n_{k}} x_{0}, f u\right) \\
\leq & \frac{\beta \alpha\left(f g u, f x_{n_{k}}\right)}{1-\beta \alpha\left(f g u, f x_{n_{k}}\right)} d\left(f x_{n_{k}-1}, f x_{n_{k}}\right)+\frac{\beta \mu\left(f u, f x_{n_{k}}\right)}{1-\beta \alpha\left(f g u, f x_{n_{k}}\right)} d\left(f x_{n_{k}}, f u\right) \\
\leq & \frac{\beta \alpha\left(f g u, f x_{n_{k}}\right)}{1-\beta \alpha\left(f g u, f x_{n_{k}}\right)}\left(\frac{\beta}{1-\beta}\right)^{n_{k}-1} d\left(f x_{0}, f x_{1}\right)+\frac{\beta \mu\left(f u, f x_{n_{k}}\right)}{1-\beta \alpha\left(f g u, f x_{n_{k}}\right)} d\left(f x_{n_{k}}, f u\right) .
\end{aligned}
$$


Proceeding the $\lim _{k \longrightarrow \infty}$, we obtain

$$
d(f g u, f u) \leq \text { constant } \times d(f u, f u),
$$

which proves that $d(f u, f u)=0$. From the triangular inequality, we have

$$
d(f u, f u) \leq \alpha(f u, u) d(f u, u)+\mu(u, f u) d(u, f u) .
$$

Suppose that $\alpha(f u, u) \leq \mu(u, f u)$, then

$$
d(f u, f u) \leq 2 \alpha(f u, u) d(f u, u) .
$$

On the other hand,

$$
\begin{aligned}
d(u, f u) & \leq \alpha(u, u) d(u, u)+\mu(u, f u) d(u, f u) \\
& \leq \frac{\alpha(u, u)}{1-\mu(u, f u)} d(u, u) .
\end{aligned}
$$

Note that if $\mu: X \times X \longrightarrow[1, \infty)$, then $1-\mu(u, f u) \leq 0$ and we get $d(u, f u)=0$. Thus, from (57), we obtain

$$
d(f u, f u)=0 .
$$

From (55) and (57), we deduce that $d(f g u, f u)=0$. To check the property $(p 1)$, i.e.,

$$
d(f g u, f u)=d(f u, f u)=d(f g u, f g u)=0 .
$$

It is easy to see that

$$
\begin{aligned}
d(f g u, f g u) & \leq \beta[d(f u, f g u)+d(f u, f g u)] \\
& =2 \beta d(f u, f g u)=0 .
\end{aligned}
$$

Thus, $f g u=f u$, since $f$ is one-to-one, $g u=u$. Therefore, $u$ is a fixed point of $g$.

Uniqueness: let $u, v$ be two fixed points of $g$, then $g u=u$ and $g v=v$. From the condition $(p 3)$, we have

$$
\begin{aligned}
& d(f v, f v) \leq d(f u, f v), \\
& d(f u, f u) \leq d(f u, f v) .
\end{aligned}
$$
get

On the other hand, using the triangular inequality, we

$$
\begin{aligned}
d(f u, f v) & =d(u, v) \\
& \leq a(u, u) d(u, u)+\mu(u, v) d(u, v) \\
& \leq \frac{a(u, u)}{1-\mu(u, v)} d(u, u) .
\end{aligned}
$$

Since $\mu: X \times X \longrightarrow[1, \infty)$, then $1-\mu(u, v) \leq 0$ and we get $d(f u, f v)=0$. Therefore, from (62) and (63), we obtain that

$$
d(f u, f v)=d(f u, f u)=d(f v, f v)=0 .
$$

Utilizing the property $(p 1)$ of the double controlled partial metric type space, we obtain $f u=f v$. Hence, $f$ is one-to-one so that $u=v$. Finally, by replacing $\left\{n_{k}\right\}$ with $\{n\}$, we conclude that $\left\{x_{n}\right\}$ converges to $u$ as $n \longrightarrow \infty$. Thus, the sequence $\left\{x_{n}\right\}$ converges to the unique fixed point $g$.
Corollary 1 (Banach contraction). Let $(X, d)$ be a complete double controlled partial metric type space by the functions $\alpha, \mu: X \times X \longrightarrow[1, \infty)$. Suppose that $f: X \longrightarrow X$ satisfies

$$
d(f x, f y) \leq \beta d(x, y)
$$

for all $x, y \in X$, where $\beta \in(0,(1 / 2))$. For $x_{0} \in X$, take $x_{n}=f^{n} x_{0}$, assuming that

$$
\sup _{m \geq 1} \lim _{i \longrightarrow \infty} \frac{\alpha\left(x_{i+1}, x_{i+2}\right)}{\alpha\left(x_{i}, x_{i+1}\right)} \mu\left(x_{i}, x_{m}\right)<\frac{1}{k}, \quad \text { where } k \in(0,1) \text {. }
$$

Furthermore, assume that for every $x \in X, \lim _{n \rightarrow \infty}$ $\alpha\left(x, x_{n}\right), \quad \lim _{n \longrightarrow \infty} \alpha\left(x_{n}, x\right), \quad \lim _{n \longrightarrow \infty} \mu\left(x, x_{n}\right), \quad$ and $\lim _{n \longrightarrow \infty} \mu\left(x_{n}, x\right)$ exist and are finite. Then, the sequence $\left\{x_{n}\right\}$ converges to some $u \in X$; moreover, if $\alpha$ and $\mu$ satisfy the following assumptions,

$$
\lim _{n \rightarrow \infty} \frac{\alpha\left(u, x_{n+1}\right)}{1-\beta \mu\left(x_{n+1}, f u\right)} \leq 0,
$$

then $f$ has a unique fixed point.

Remark 1. Results presented in this manuscript generalize, enrich, and theorize the prominent results due to Kannan [24] and Bojor [25] in the framework of double controlled partial metric type spaces.

Example 2. Let $X=\{0,1,2\}$; consider the function $d$ given as follows: (Table 2)

Given $\alpha, \mu: X \times X \longrightarrow[1, \infty)$ is defined as

$$
\begin{aligned}
& \alpha(x, y)=d(x, y)+5, \\
& \mu(x, y)=d(x, y)+7 .
\end{aligned}
$$

It is easy to verify that given $d$ equipped with $X$ is double controlled partial metric type space but not double controlled metric type space because $d(x, x) \neq 0$ for all $x \in X$. Now, we define a mapping $f: X \longrightarrow X$ by the following:

$$
f(x)= \begin{cases}1, & \text { when } x=\{1,2\}, \\ 2, & \text { when } x=0 .\end{cases}
$$

Choose $f 0=2$ and $f 2=1$, then by using (19), we acquire

$$
\begin{aligned}
d(f 0, f 2) & \leq \beta[d(0, f 0)+d(2, f 2)] \\
d(2,1) & \leq \beta[d(0,2)+d(2,1)] \\
\frac{1}{5} & \leq \beta\left(\frac{2}{7}+\frac{1}{5}\right) \\
\frac{1}{5} & \leq \beta\left(\frac{17}{35}\right) .
\end{aligned}
$$

Since $\beta \in(0,(1 / 2))$, we choose $\beta=(8 / 17)$; taking $x_{0}=0$ and $k=(1 / 8)$, it is clear that condition (20) is satisfied as follows: 
TABLe 2: Metric $d$ defined in Example 2.

\begin{tabular}{cccc}
\hline$d$ & 0 & 1 & 2 \\
\hline 0 & $1 / 27$ & $1 / 4$ & $2 / 7$ \\
1 & $1 / 4$ & $1 / 28$ & $1 / 5$ \\
2 & $2 / 7$ & $1 / 5$ & $1 / 29$ \\
\hline
\end{tabular}

$$
\sup _{m \geq 1} \lim _{i \longrightarrow \infty} \frac{\alpha\left(x_{i+1}, x_{i+2}\right)}{\alpha\left(x_{i}, x_{i+1}\right)} \mu\left(x_{i}, x_{m}\right)=\frac{1768}{245}<8=\frac{1}{k} .
$$

Since inequality (20) is satisfied for every $x \in X$, additionally, for each $x \in X$, we have

$$
\begin{aligned}
& \lim _{n \longrightarrow \infty} \alpha\left(x, x_{n}\right)=\max (0, x)<\infty, \\
& \lim _{n \longrightarrow \infty} \alpha\left(x_{n}, x\right)=\max (x, 0)<\infty, \\
& \lim _{n \longrightarrow \infty} \mu\left(x, x_{n}\right)=\max (0, x)<\infty, \\
& \lim _{n \longrightarrow \infty} \mu\left(x_{n}, x\right)=\max (x, 0)<\infty .
\end{aligned}
$$

Therefore, all the hypotheses of Theorem 1 are contended and 1 is the unique fixed point of $f$.

\section{Conclusions}

We launched a new concept of double controlled partial metric type spaces which expands the ideas of certain variants of metric spaces, viz., controlled metric type spaces, double controlled metric type spaces, and partial metric spaces. The introduced results sum up and broaden some previous writing, and some illustrative examples are investigated to show the potency of our work.

\section{Data Availability}

No data were used to support this study.

\section{Conflicts of Interest}

The authors declare that they have no conflicts of interest.

\section{References}

[1] A. Bakhtin, "The contraction mapping principle in quasimetric spaces," vol. 30, pp. 26-37, Functional Analysis, 1989.

[2] S. Czerwik, "Contraction mappings in b-metric spaces," Acta Mathematica et Informatica Universitatis Ostraviensis, vol. 5-11, 1993.

[3] T. Kamran, M. Samreen, and Q. Ul Ain, "A generalization of b-metric space and some fixed point theorems," Mathematics, vol. 5, no. 19, p. 2, 2017.

[4] M. Younis, D. Singh, I. Altun, and V. Chauhan, "Graphical structure of extended b-metric spaces: an application to the transverse oscillations of a homogeneous bar," International Journal of Nonlinear Sciences and Numerical Stimulation, 2021.

[5] M. Younis, D. Singh, and L. Shi, "Revisiting graphical rectangular b-metric spaces," Asian-European Journal of Mathematics, 2021.
[6] T. Abdeljawad, N. Mlaiki, H. Aydi, and N. Souaya, "Double controlled metric type spaces and some fixed point results," Mathematics, vol. 6, no. 12, p. 320, 2019.

[7] N. Mlaiki, H. Aydi, N. Souayah, and T. Abdeljawad, "Controlled metric type spaces and the related contraction principle," Mathematics, vol. 6, no. 10, p. 194, 2018.

[8] N. Souayah and M. Mrad, "On fixed-point results in controlled partial metric type spaces with a graph," Mathematics, vol. 8, no. 1, p. 33, 2020.

[9] S. Radenovic, T. Došenovic, T. A. Lampert, and Z. Golubovíc, "A note on some recent fixed point results for cyclic contractions in b-metric spaces and an application to integral equations," Applied Mathematics and Computation, vol. 273, pp. 155-164, 2016.

[10] S. Radenovic, K. Zoto, N. Dedovic, V. Šešum-Cavic, and A. H. Ansari, "Bhaskar-Guo-Lakshmikantam-Ciric type results via new functions with applications to integral equations," Applied Mathematics and Computation, vol. 357, pp. 75-87, 2019.

[11] A. Shoaib, S. S. Alshoraify, and M. Arshad, "Double controlled dislocated quasi-metric type spaces and some results," Journal of Mathematics, vol. 2020, Article ID 3734126, 8 pages, 2020.

[12] A. Shoaib, S. S. Alshoraify, P. Kumam, S. Saleh Alshoraify, and M. Arshad, "Fixed point results in double controlled quasi metric type spaces," AIMS Mathematics, vol. 6, no. 2, pp. 1851-1864, 2021.

[13] M. Younis, D. Singh, and A. Goyal, "A novel approach of graphical rectangular b-metric spaces with an application to the vibrations of a vertical heavy hanging cable," Journal of Fixed Point Theory and Applications, vol. 21, p. 33, 2019.

[14] M. Younis, D. Singh, S. Radenovic, and M. Imdad, "Convergence theorems via generalized contractions and its applications," Filomat, vol. 34, no. 3, 2020.

[15] M. Younis and D. Singh, "On the existence of the solution of Hammerstein integral equations and fractional differential equations," Journal of Applied Mathematics and Computing, 2021.

[16] S. G. Matthews, Partial Metric Spaces, Department of Computer Science, University of Warwick, Coventry, England, 1992.

[17] S. G. Matthews, "Partial metric topology," Annals of the New York Academy of Sciences, vol. 728, pp. 183-197, 1994.

[18] F. Gu and W. Shatanawi, "Some new results on common coupled fixed points of two hybrid pairs of mappings in partial metric spaces," Journal of Nonlinear Functional Analysis, vol. 2019, Article ID 13, 2019.

[19] L. V. Nguyen and N. T. N. Tram, "Fixed point results with applications to involution mappings," Journal of Nonlinear Variable Analysis, vol. 4, pp. 415-426, 2020.

[20] K. Javed, H. Aydi, F. Uddin, and M. Arshad, "On orthogonal partial-metric spaces with an application," Journal of Mathematics, vol. 2021, Article ID 6692063, 7 pages, 2021.

[21] H. Aydi, E. Karapinar, and W. Shatanawi, "Coupled fixed point results for $(\psi, \varphi)$-weakly contractive condition in ordered partial metric spaces," Computers and Mathematics with Applications, vol. 62, no. 12, pp. 4449-4460, 2011.

[22] E. Karapinar, R. Agarwal, and H. Aydi, "Interpolative reichrus-cirić type contractions on partial metric spaces," Mathematics, vol. 6, no. 11, p. 256, 2018.

[23] F. Vetro, "Common fixed points of mappings satisfying implicit relations in partial metric spaces," The Journal of Nonlinear Science and Applications, vol. 6, no. 3, pp. 152-161, 2013. 
[24] R. Kannan, "Some results on fixed points," Bulletin of the Calcutta Mathematical Society, vol. 10, pp. 71-76, 1968.

[25] F. Bojor, "Fixed points of Kannan mappings in metric spaces endowed with a graph," "Analele Universitatii" "Ovidius" " Constanta-Seria Matematica”, vol. 20, no. 1, pp. 31-40, 2012. 